\title{
Extracellular Receptor Kinase and cAMP Response Element Binding Protein Activation in the Neonatal Rat Heart after Perinatal Cocaine Exposure
}

\author{
LENA S. SUN AND AARON QUAMINA \\ Department of Anesthesiology [L.S.S., A.Q.] and Pediatrics [L.S.S.], College of Physicians \& Surgeons, \\ Columbia University, New York, NY 10032
}

\begin{abstract}
Prenatal exposure to cocaine has been shown to induce an increase in the myocardial expression and activation of the cAMP response binding protein (CREB), a transcriptional factor that has been shown to regulate gene expression. Several different kinases, including protein kinase A, calcium calmodulin kinase II, and mitogen-activated protein kinase can induce phosphorylation of CREB at serine 133, a necessary step for CREB activation. We examined whether the mitogen-activated protein kinase-extracellular receptor kinase (ERK) pathway may be involved in mediating the serine 133 CREB phosphorylation in cardiac nuclei after perinatal cocaine exposure. Pregnant rats were treated daily with saline or cocaine at $60 \mathrm{mg} / \mathrm{kg}$ (C60) by intragastric administration during the entire gestational period, and treatment was continued in the nursing dams after delivery until the time of the study. Nuclear extracts were isolated from hearts of 1-d- and 7-d-old neonatal rats. We performed immunoblotting experiments using an antibody that recognized CREB with phosphorylation specifically at the serine 133 site and an antibody that recognized both the phosphorylated and the unphosphorylated forms of CREB, as well as antibodies for total ERK, phospho-ERK, total ribosomal S6 kinase 1 (RSK1), RSK2,
\end{abstract}

\section{ABSTRACT}

and phospho-RSK. We assessed the interaction of RSK with CREB or CREB-binding protein by performing co-immunoprecipitation experiments. We found that perinatal cocaine exposure increased both phospho-ERK and phospho-RSK expression, indicative of an increased activity of these two enzymes. Furthermore, we demonstrated that phospho-RSK was immunoprecipitated with CREB in all neonatal cardiac nuclei and that the greatest interaction was found in day 7 hearts after perinatal cocaine exposure. Our results thus illustrate that the ERK-RSK pathway was active in the postnatal rat heart at 1 and $7 \mathrm{~d}$ of age and that this pathway may mediate the increase in myocardial CREB activation after perinatal cocaine exposure in the day 7 hearts. (Pediatr Res 56: 947-952, 2004)
CBP, CREB-binding protein
CREB, cAMP response element binding protein
ERK, extracellular receptor kinase
MAPK, mitogen-activated protein kinase
PKA, protein kinase A
RSK, ribosomal S6 kinase

\section{Abbreviations}

We have previously shown that perinatal cocaine exposure increased the expression of the phosphorylated cAMP response element binding protein (CREB), the activated form of this transcriptional factor, in the 1-d and 7-d-old neonatal rat heart. The increased expression of phospho-CREB, detected by an antibody that specifically recognized CREB that was phosphorylated at serine 133, corresponded to an increase in CRE binding activity (1). Because we found protein kinase A (PKA)

Received November 17, 2003; accepted June 7, 2004.

Correspondence: Lena S. Sun, M.D., College of Physicians \& Surgeons, Columbia University, Department of Anesthesiology and Pediatrics, 630 West 168th Street, BH4440 North, New York, NY 10032; e-mail: 1ss4@ columbia.edu

The study was support in part by the Department of Health and Human Services, NIDA RO1 DA12962 (L.S.S.).

DOI: 10.1203/01.PDR.0000145279.42838.34 activity to be greater in cocaine-exposed 1-d-old rat hearts, this pathway may be one possible mechanism for the up-regulation in phospho-CREB expression at day 1 (1). In contrast, there was no change in PKA activity in cocaine-exposed neonatal rat hearts at day 7. Therefore, we concluded that non-PKAdependent pathways must also be involved in the phosphorylation of CREB in the neonatal rat heart after perinatal cocaine exposure, particularly at day 7 .

Mitogen-activated protein kinase (MAPK) is one of the signaling pathways that has been shown to mediate serine 133 phosphorylation in cell lines and neuronal tissues (2-6). Members of the MAPK family include extracellular receptor kinases (ERK1 and ERK2), p38 MAPK and Jun kinases. A substrate of ERK is the family of p90 ribosomal S6 kinase (RSK), which comprises three isoforms, RSK1, RSK2, and RSK3 $(7,8)$. In 
response to growth factor stimulation, CREB phosphorylation at serine 133 was shown to occur via coupling of ERK and nuclear p90 RSK in vascular smooth muscles (9). CREB activation via the MAPK pathway in response to nerve growth factor and epidermal growth factor stimulation has been shown to involve RSK2 as the CREB kinase (10). In isolated cardiomyocytes, stimulation of the MAPK/RSK pathway, including Raf-1, MAPKs, and p90 ${ }^{\text {rsk }}$, led to the activation of ATF-2 and induction of jun-B mRNA (11-13). Whereas ERK/RSK signaling pathway seems to be functional in cardiomyocytes, the precise role of the ERK-RSK signaling pathway in CREB phosphorylation in cardiac tissues has not been studied. In the present study, we sought to determine whether the ERK-RSK pathway might be involved in CREB phosphorylation in neonatal rat hearts after perinatal cocaine exposure.

\section{METHODS}

Materials and reagents. ERK2 (C-14), phospho-Y204 ERK (E4), phospho-RSK, RSK1 (C21), and RSK2 (C19) specific antibodies were obtained form Santa Cruz Biotechnologies. CREB and phospho-S133 CREB antibodies were obtained from Cell Signaling Technologies.

Animal model. Studies were approved by the Institutional Animal Care and Use Committee at the College of Physicians and Surgeons of Columbia University. Timed-pregnant (gestational day 0-1) female Sprague-Dawley rats that were 90$110 \mathrm{~d}$ of age were purchased and housed individually in a temperature/humidity-controlled room on a 12/12-h light/dark cycle with ad libitum access to Purina laboratory rodent chow and drinking water. Upon arrival in our animal care facility, these rats were weighed, then transferred to a maternal cage within the first $24 \mathrm{~h}$. Weights were obtained on days 7, 14, and 21 of gestation.

Saline (control) and cocaine at $60 \mathrm{mg} / \mathrm{kg}$ (C60) in equal volume was given once a day beginning on gestational day 2 until birth by intragastric administration. These doses and the intragastric route of administration have been documented to simulate the plasma and tissue concentrations achieved in the human abusers. After parturition, postpartum cocaine-treated mothers continued to receive cocaine as before until postpartum day 7. Neonatal animals remained with their biologic mothers and were nursed by them. Animals were killed on day 1 or day 7 of postnatal life. Animals that were used for the experiments at day 1 and day 7 were obtained from a minimum of four litters in all experiments. Tissues from animals were harvested from different litters for each individual experiment.

Tissue preparation and fractionation. Neonatal rat ventricles were isolated and either immediately frozen in liquid nitrogen for storage or homogenized on ice using Dounce homogenizer in ice-cold Buffer A (10 mM of HEPES, $10 \mathrm{mM}$ of $\mathrm{KCl}, 1.5 \mathrm{mM}$ of $\mathrm{MgCl}_{2}, 1 \% \quad \beta$-Mercaptoethanol, $20 \%$ glycerol, and Roche Biochemicals protease inhibitor cocktail). Homogenate was spun at $10,000 \times g$ for $5 \mathrm{~min}$ at $4^{\circ} \mathrm{C}$. The supernatant was saved as nonnuclear fraction. The pellet was resuspended in 10 volumes of ice cold Buffer A. Suspension was centrifuged again, and the supernatant was discarded. The pellet was resuspended in 3 volumes of ice-cold Buffer B (20
$\mathrm{mM}$ of HEPES, $1.5 \mathrm{mM}$ of $\mathrm{MgCl}_{2}, 1 \% \beta$-Mercaptoethanol, $25 \%$ glycerol, $2 \mathrm{mM}$ of EDTA, $0.5 \mathrm{M}$ of $\mathrm{NaCl}, 0.1 \%$ Triton $\mathrm{X}-100$, and Roche Biochemicals protease inhibitor cocktail). Buffer B suspension was incubated on ice for 30 min with occasional mixing then centrifuged at $15,000 \mathrm{rpm}$ in a tabletop Microfuge for $15 \mathrm{~min}$ at $4^{\circ} \mathrm{C}$. The nuclear protein-enriched supernatant was collected for Western blot analysis. The integrity of both nuclear and nonnuclear extracts was assessed by Western blot analysis. Nuclear fractions were enriched for histone compared with crude total heart lysates. In nonnuclear fractions, histone was absent. The histone-positive nuclear fractions were used in subsequent studies (1).

Western blots. Nuclear and nonnuclear fractions were diluted 1:1 in Laemlli's loading buffer. Diluted samples were heat denatured. Samples with equivalent protein, as determined by Bradford's assay, were loaded onto Invitrogen Xcell SDSPAGE/Western blotting module. Proteins were resolved on either a $10 \%$ or a $4-20 \%$ gradient. Proteins were transferred to polyvinylidene diflouride membrane (Bio-Rad) at $20 \mathrm{~V}$, either overnight at $4^{\circ} \mathrm{C}$ or for $2 \mathrm{~h}$ at room temperature. Membranes were briefly washed in PBS-Tween ( $1 \times$ PBS, $0.1 \%$ Tween 20$)$ then blocked for $1 \mathrm{~h}$ in $5 \%$ nonfat milk/PBS-Tween at room temperature. Membranes were then incubated overnight at $4^{\circ} \mathrm{C}$ in primary antibody diluted in $1 \%$ nonfat milk/PBS-Tween as previously described. The next day, membranes were washed 3 $\times 10 \mathrm{~min}$ in PBS-Tween. Membranes were then incubated for $1 \mathrm{~h}$ at room temperature, in secondary antibody-horseradish peroxidase conjugate diluted in $1 \%$ nonfat milk/PBS-Tween. Membranes were then washed three times as before. Proteins were visualized by chemiluminescence using Amersham ECLPlus reagent according to the manufacturer's instructions.

Immunoprecipitation. All reagents for these reactions were maintained at or below $4^{\circ} \mathrm{C}$. A total of $200 \mu \mathrm{g}$ of nuclear protein was incubated with anti-CREB or anti-CREB-binding protein (anti-CBP) antibody and IP buffer (20 mM HEPES, 1 $\mathrm{mM}$ EDTA, $0.2 \%$ NP-100, $100 \mu \mathrm{g} / \mathrm{mL}$ BSA, and Roche Biochemicals protease inhibitor cocktail). Reactions were allowed to proceed overnight at $4^{\circ} \mathrm{C}$. The next day, $50 \mu \mathrm{L}$ of protein A/G-agarose suspension was added to immunoreactions. These reactions were allowed to proceed for $1 \mathrm{~h}$ at $4^{\circ} \mathrm{C}$. After $1 \mathrm{~h}$, protein A/G-agarose beads were spun at 10,000 $\times g$ for $15 \mathrm{~s}$. Beads were then washed three times in $1 \mathrm{~mL}$ of ice-cold PBS/1\%Tween. After final wash, supernatant was removed, pellets were spun again, and residual fluid was removed. Pellets were then resuspended and boiled in SDS loading buffer. Equivalent volumes of samples were then loaded onto $10 \%$ SDS polyacrylamide gel for Western blot analysis using an anti-RSK1, anti-RSK2, or anti-pRSK antibody.

Data analysis. All data presented are based on analysis performed in nuclear fractions. Differences within each age between control and cocaine-treated groups were analyzed by unpaired $t$ test because there were only two groups. We used ANOVA to determine differences between day 1 and day 7 within each treatment group. We estimated a sample size of five on the basis of power that used an $\alpha$ value of 0.05 and a power of 0.8 to detect a $25 \%$ difference between groups. Results were deemed statistically significant at $p<0.05$. 


\section{RESULTS}

A representative blot of histone immunoblot in neonatal hearts from day 1 and day 7 neonatal rats is shown to document the successful separation of nuclear and nonnuclear fractions (Fig. 1). Histone is used because it is a nuclear-specific protein. Figure 1 further illustrates the equivalent loading of nuclear proteins from all of the samples in question.

Perinatal cocaine exposure and ERK activation in the neonatal rat heart. In neonatal rat hearts, we investigated whether MAPK pathway may be differentially activated after perinatal cocaine exposure. In the day 1 and day 7 rat hearts, immunodetectable unphosphorylated ERK 42 and ERK 44 were present in both the nuclear and nonnuclear fractions (Fig. $2 A$ ). There were no apparent differences between saline (control) and cocaine-exposed (C60) neonatal hearts with respect to the abundance of nuclear ERK 42 or ERK 44 at day 1 or day 7 , but cocaine exposure induced an increase in nonnuclear ERK 42 and ERK 44 at day 7 and ERK 44 at day 1. Using a phospho-specific antibody that recognized ERK phosphorylated at tyrosine 204 (P-ERK), we found phospho-ERK expression to be present in both nuclear and cytosolic fractions. However, only nuclear phospho-ERK expression seemed to be significantly increased in cocaine-exposed day 1 and day 7 hearts (Fig. $2 B$ and $C$ ). Moreover, nuclear p-ERK 42 was much more prominent than p-ERK 44 in both day 1 and day 7 hearts. Using phospho-ERK expression as a means to assess ERK activation, our findings are consistent with the idea that perinatal cocaine exposure was associated with enhanced ERK activation in the neonatal heart. Although there was a significant increase in ERK activation in both day 1 and day 7 hearts, the enhanced ERK activation was much greater in day 7 hearts $(107 \pm 23.5$ and $118 \pm 36 \%$ above control for pERK42 and pERK44, respectively) than in day 1 hearts $(51 \pm 26$ and $45 \pm$ $17 \%$ above control for pERK42 and pERK44, respectively).

RSK localization and activation. One of the downstream effectors of the ERK signaling cascade is the p90 RSK. After translocation of RSK from the cytoplasm into the nucleus, it becomes activated via phosphorylation by the extracellular signal-regulated kinases and thereby acts to effect specific changes in gene expression, cell-cycle regulation, and protein synthesis regulation. In the neonatal heart, both RSK1 and RSK2 are detectable in nuclear and nonnuclear fractions (Fig. 3 ), with the great majority of both RSK1 and RSK2 being present in the nonnuclear fraction. Although little difference was evident in nuclear RSK2 between saline (control) and

\begin{tabular}{|c|c|c|c|c|c|}
\hline \multicolumn{2}{|c|}{ Day One } & \multicolumn{4}{|c|}{ Day Seven } \\
\hline Nuclear & Non-nuclear & & ear & Nor & Iclear \\
\hline $\mathrm{C} 60$ & CTL C60 & CTL & $\mathrm{C} 60$ & CTL & $\mathrm{C} 60$ \\
\hline
\end{tabular}

Figure 1. A representative immunoblot of histone is shown in nuclear and nonnuclear fractions in from day 1 and day 7 neonatal rat hearts. Histone was found exclusively in the nuclear fraction, and no histone was evident in the nonnuclear fractions. Comparable amounts of histone was detected in control (CTL) and cocaine-treated (C60) hearts at day 1 and day 7.

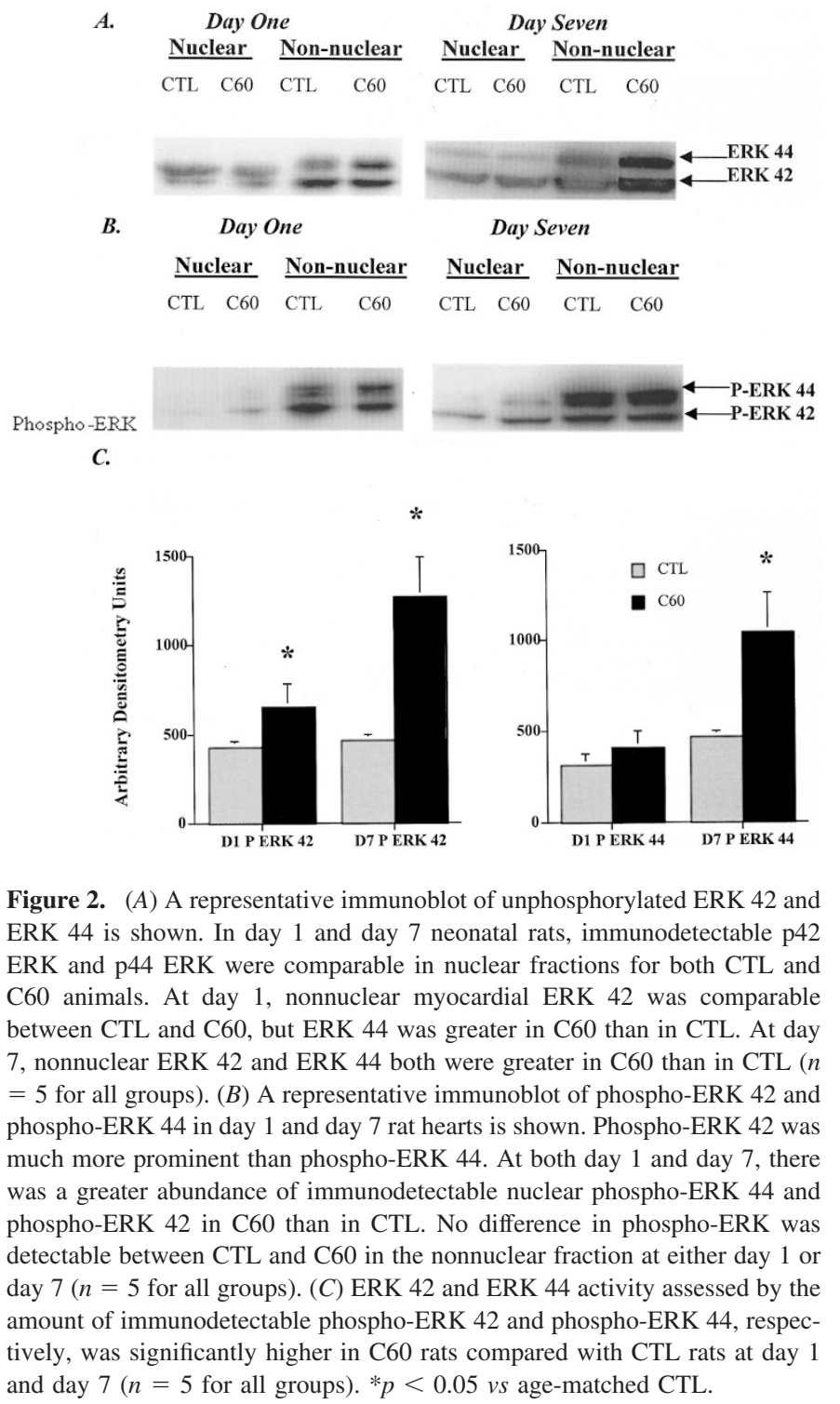

cocaine-exposed (C60) neonatal hearts at both ages, nuclear RSK1 was significantly greater in C60 hearts at day 1 compared with any of the other groups (Fig. 2). Using a phosphospecific antibody directed against RSK that is phosphorylated at serine ${ }^{380}$, phospho-RSK expression was greatly enhanced by perinatal cocaine exposure in both day 1 and day 7 hearts (Fig. 4). In the nonnuclear fraction, an additional prominent upper band was observed in both control and C60 at both ages. This band could represent RSK phosphorylation at other sites.

Cocaine-induced changes in protein association. To investigate further the relationship between the increased ERK/RSK activity and up-regulation in phospho-CREB expression in response to perinatal cocaine exposure, we performed immunoprecipitation experiments to examine the association of RSK and CREB in the neonatal myocardial tissues. Neonatal rat cardiac nuclear extracts were first immunoprecipitated with an anti-CREB antibody, then subjected to immunoblotting with p-RSK-, RSK1-, or RSK2-specific antibodies. An increased pRSK expression was seen in the CREB-immunoprecipitated cardiac nuclei isolated from cocaine-exposed animals at day 7 
Day One

Day Seven

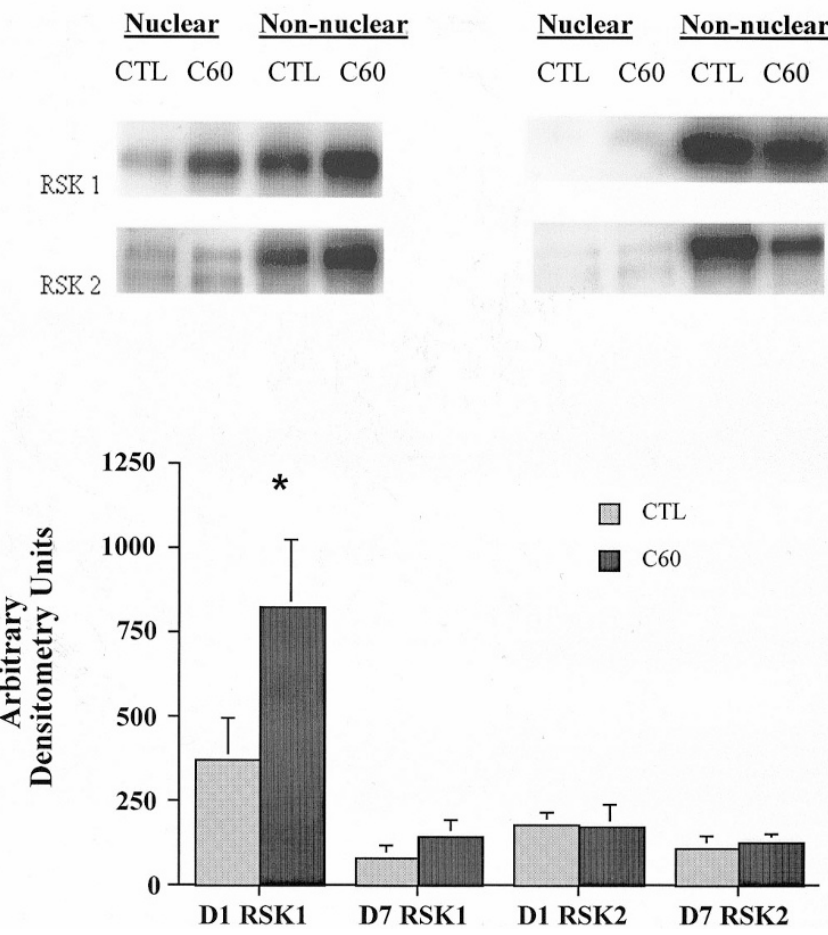

Figure 3. The graph illustrates the amount of immunodetectable nuclear RSK1 and RSK2 for day 1 (D1) and day 7 (D7) neonatal rats with perinatal exposure to cocaine (C60) or saline (CTL). RSK1 was significantly greater in D1 C60 than in D1 CTL but was comparable at D7. RSK2 was similar between CTL and C60 for both D1 and D7. The immunoblot is a representative gel illustrating these results. In addition, the amount of immunodetectable nonnuclear RSK1 and RSK2 is shown. * $p<0.05 v s$ age-matched CTL.

( $n=5 p<0.05$; Fig. 5 ) but not at day 1 ( $n=5$; Fig. 4). Our results thus suggest that there was an increase in CREB-pRSK interaction at day 7 after perinatal cocaine exposure, consistent with the ERK-RSK pathway playing a role in mediating the increase in phosphorylation of CREB observed. We were able to demonstrate that both RSK1 and RSK2 were coimmunoprecipitated with CREB in the day 1 and day 7 cardiac nuclear extracts, but there were no detectable differences in RSK1-CREB or RSK2-CREB interaction between control and cocaine-treated animals at either age (data not shown). These results suggest that whereas RSK-CREB interaction probably involves both RSK1 and RSK2, the increase in pRSK-CREB interaction cannot be attributed to changes in the total amount of either isoform of RSK that is bound to CREB. Because CBP, a coactivator of CREB binding, has been shown to modulate RSK-CREB interaction negatively, we also performed immunoprecipitation experiments to examine whether perinatal cocaine exposure influenced the interaction between RSK and CBP. CBP-immunoprecipitated cardiac nuclei were subjected to immunoblotting with RSK1 or RSK2 antibodies. There were no differences detected between control and cocaine-treated animals at either age (data not shown). These results support the idea that cocaine-induced changes in CREB activation was not the result of an indirect modulation by CBP via RSK interaction. Furthermore, these findings are consistent with a direct role for the MAPK-RSK pathway in the activation of CREB.
Day One

Nuclear Non-nuclear

CTL C60 CTL C60

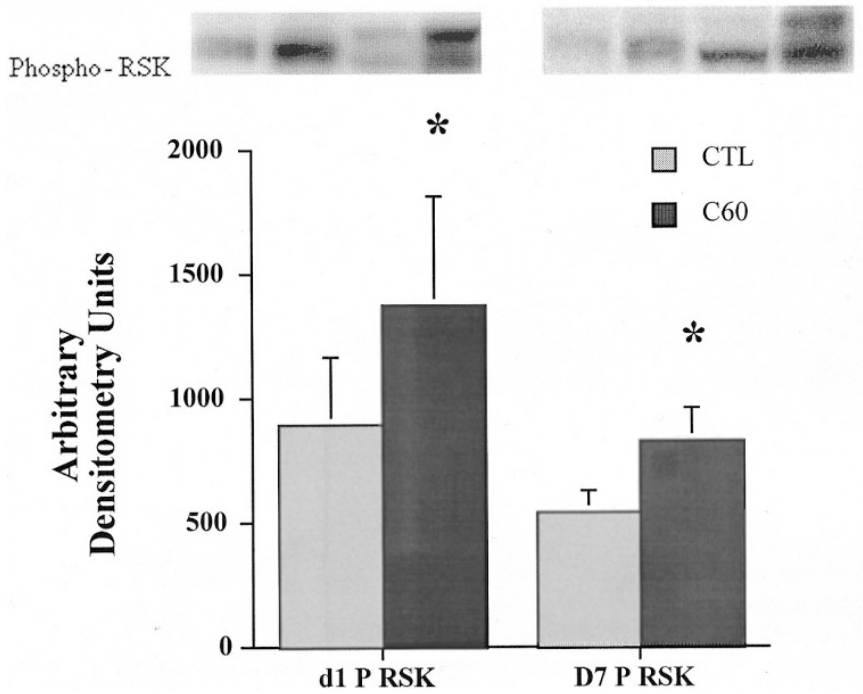

Figure 4. Immunodetectable cardiac nuclear phospho-RSK was significantly greater in neonatal rats with perinatal exposure to cocaine (C60) than to saline (CTL) at $1 \mathrm{~d}$ and $7 \mathrm{~d}$ of age. A representative immunoblot is shown illustrating the increased phospho-RSK expression in C60 hearts in both nuclear and nonnuclear fractions for both day 1 and day 7 animals ( $n=5$ for all groups). $* p<0.05$ vs age-matched CTL.

\section{DISCUSSION}

The major findings of our study are as follows: 1) there was a concomitant increase in both phospho-CREB expression and the activity of the ERK-RSK signaling pathway in the neonatal rat heart after perinatal cocaine exposure, and 2) we demonstrated that there was an interaction between RSK and CREB in the neonatal rat heart. Taken together, these results indicate that the ERK-RSK signaling pathway may mediate CREB phosphorylation in the neonatal rat heart.

Neurotransmitters, growth factors, and hormones induce CREB activation through serine 133 phosphorylation, which can be mediated via cAMP/calcium-dependent kinase signaling pathways (14). They include 1) cAMP-dependent PKA $(15), 2)$ protein kinase $\mathrm{C}(16), 3)$ calcium-calmodulin kinase II and IV $(17,18), 4)$ MAPK ERK-activated p90RSK $(19,20), 5)$ p38 MAPK-activated protein kinase 2 (20-23), and 6) PI-3 kinase activated Akt/PKB (24). Most of the studies examining signal transudation pathways for CREB phosphorylation have been performed in cells in vitro $(14,25)$. PKA-mediated CREB phosphorylation is the most predominant mechanism, but many studies have also documented that MAPK signaling pathway can play a role in the activation of CREB $(15,22,26)$. In oligodendrocytes, Ca-dependent activation of MAPK pathway induces serine 133 CREB phosphorylation (27). In vascular smooth muscle cells, activation of ERK-RSK has been shown to induce CREB phosphorylation (28). In response to growth factor stimulation, the CREB kinase activated by the RAS-MAPK pathway was found to be RSK2 (10). RSK has also been identified as the probable CREB serine 133 kinase in 

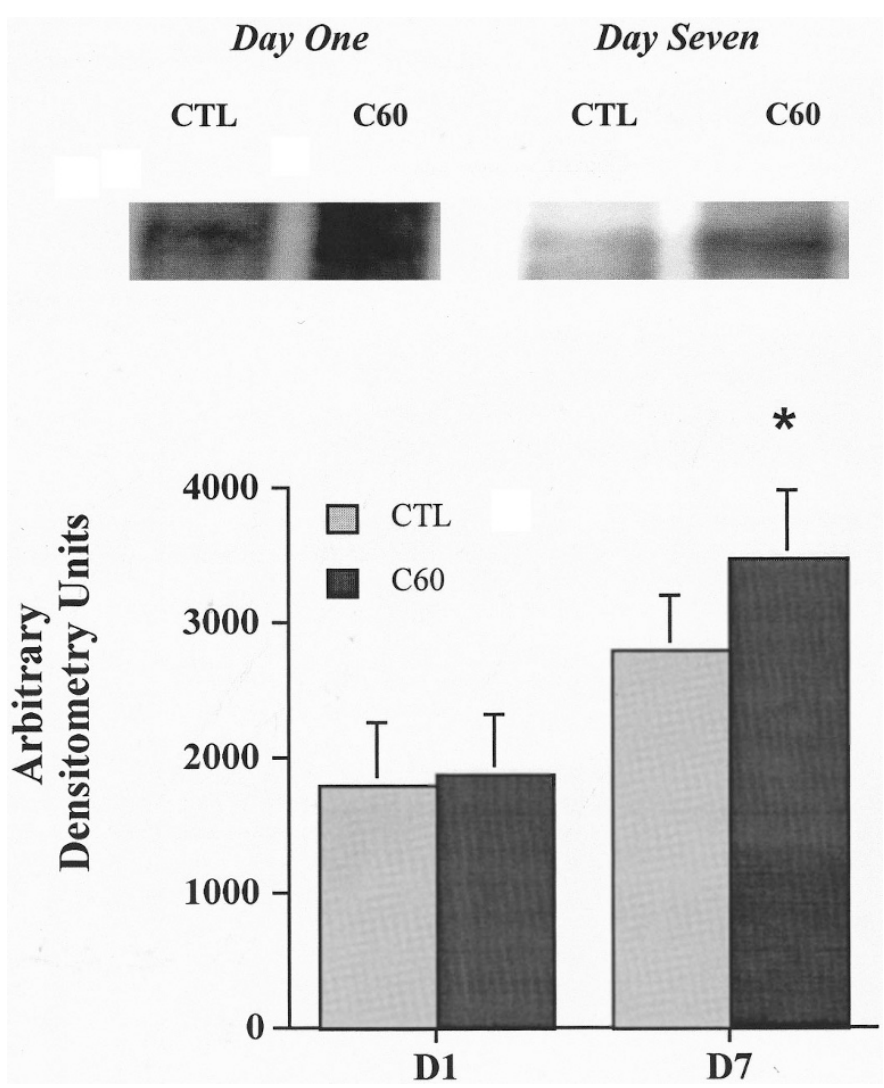

Figure 5. Neonatal rat cardiac nuclei were immunoprecipitated with an anti-CREB antibody and then subjected to immunoblotting with an antibody specific for phospho-RSK. There was a demonstrable CREB-phospho-RSK association in all groups. There was no difference in CREB-phospho-RSK association between CTL and C60 rats at D1 $(n=5)$. In contrast, CREBphospho-RSK association was much greater in C60 than in CTL cardiac nuclei at $\mathrm{D} 7(n=5)$. A representative immunoblot is shown. ${ }^{*} p<0.05 v \mathrm{~s}$ age-matched CTL.

vivo and in vitro $(10,29)$. A functional MAPK-RSK pathway has been shown to exist in cardiomyocytes under a variety of conditions (11-13,30). Activation of the ERK-RSK pathway has been documented in neonatal cardiomyocytes after stimulation by arginine vasopressin (AVP) (13) and vascular endothelial growth factors (12). The downstream effector activated by the ERK-RSK signaling pathway in cardiomyocytes, however, was not specifically examined. Whether this signaling pathway might play a role in the activation of CREB has not been determined.

In the present study, we demonstrated that perinatal exposure to cocaine stimulated phosphorylation and activation of the MAPK ERK signaling pathway. We further demonstrated that perinatal cocaine exposure was associated with an increase in activation and phosphorylation of RSK, suggesting that the ERK-RSK signaling pathway was up-regulated in neonatal hearts after cocaine exposure. An increase in ERK activity has been shown to increase phospho-RSK expression. Upon activation, ERK associates with RSK to induce phosphorylation at serine $^{369}$ and tyrosine $e^{570}$, two sites found in the $\mathrm{C}$ terminus kinase domain of the RSK protein $(7,8)$. This leads to the removal of inhibition of RSK autophosphorylation at the C terminus. Autophosphorylation then proceeds at multiple sites at the $\mathrm{C}$ terminus and the $\mathrm{N}$ terminus kinase domains, which eventually leads to the full activation of RSK. Phospho-RSK expression can also be modified secondary to changes in the total amount of nuclear RSK available for phosphorylation. We determined the effect of perinatal cocaine exposure on the total amount of the two isoforms of RSK in the nucleus and the cytosol. Cocaine did not affect the expression of nuclear or cytosolic RSK2, the isoform shown to be the CREB kinase that mediates CREB phosphorylation in response to growth factor stimulation. In contrast, both nuclear localization of RSK1 and cytosolic fraction of RSK1 were increased at day 1 but not at day 7 in the cocaine-exposed hearts. Therefore, the cocaineinduced increase in phospho-RSK expression at day 1 may be due to an increase in phosphorylation of the RSK1 isoform. It has been reported that RSK1 but not RSK2 expression is developmentally regulated in the heart, with a $50 \%$ postnatal decrease in RSK1 expression by $50 \mathrm{~d}$ of age (31). Therefore, in the present study, the increase in phospho-RSK at day 1 might be attributable to the relatively greater abundance of RSK1 isoform immediately after birth at day 1 . RSK phosphorylation may also increase or decrease as result of changes in the recruitment of RSK to CBP $(7,32)$. Persistent ERK activation has been shown to decrease CREB activity (i.e. decreased phospho-CREB expression) via an increase in CBP-RSK association, resulting in hypophosphorylation of RSK (33). The converse situation of hyperphosphorylation of RSK in the face of decreased CBP-RSK association has not been documented. However, a possible alternative mechanism for the increased phospho-CREB expression in our model might occur via reduced CBP-RSK interaction, leading to an increase in RSK phosphorylation. This explanation was unlikely because our results did not show any difference in CBP-RSK association between cocaine and control hearts. Therefore, the increase in phospho-RSK expression was most likely due to a direct effect of enhanced ERK activity and an increase in total nuclear RSK1 in the case of day 1 hearts. Our study used a phosphoRSK antibody that specifically recognized phosphorylation at the extreme $\mathrm{C}$ terminus serine site, but it did not distinguish among the three different isoforms of RSK. We chose to examine only RSK1 and RSK2 because RSK3 activity has been shown to not be unaffected by in vitro incubation with ERK1 or ERK2 (8). The increase in phospho-RSK expression in our study may be due to a concomitant increase in phosphorylation of both RSK1 and RSK2 isoforms or of only one isoform. In both day 1 and day 7 hearts, cocaine exposure did not increase RSK2 expression but significantly increased the nuclear and nonnuclear expression of RSK1 at day 1. It is tempting to speculate that the up-regulation in phospho-RSK observed in our study may reflect a selective increase in phosphorylation of the RSK1 isoform in day 1 hearts but may be less isoform specific in day 7 hearts.

We also demonstrated that phospho-RSK can be coimmunoprecipitated with CREB in both control and cocaineexposed hearts, thus confirming a direct interaction of phospho-RSK and CREB. The interaction between phospho-RSK and CREB, however, was significantly augmented only in hearts from day 7 animals after perinatal cocaine exposure. We did not find any difference in RSK1-CREB or RSK2-CREB 
interaction in control or cocaine hearts at either age. These results are consistent with the idea that the up-regulation in phospho-CREB expression was mediated via direct phosphorylation of serine 133 by phospho-RSK, the activated form of RSK.

We have previously shown that PKA activity was enhanced in the day 1 neonatal hearts with prenatal cocaine exposure, but the up-regulation in PKA activity was not evident in day 7 hearts (1). We have now investigated whether the MAPK/RSK signaling pathway may be involved in the phosphorylation of CREB in the neonatal heart. Our results indicate that this pathway may play an important role in increasing CREB activation in the neonatal rat heart, in the context of perinatal cocaine exposure. Whereas cocaine exposure stimulated both PKA and MAPK/RSK pathway at day 1, there was no evidence that phospho-RSK-CREB association was different between cocaine and control hearts. In response to perinatal cocaine exposure, there was a more robust ERK activation and CREBpRSK interaction at day 7. Taken together, these results suggest that PKA may be more predominant at day 1 and MAPK/ RSK may be more significant at day 7 in increasing myocardial CREB activity after cocaine exposure. Earlier studies indicated that there may be differences in CREB activity depending on the specific signaling pathway used to induce CREB phosphorylation. Target gene activation may be discriminated on the basis of the kinase signaling pathway inducing CREB phosphorylation (14,34). At the promoter level, PKA-mediated CREB phosphorylation can activate target genes via a single CRE consensus region. MAPK-mediated CREB phosphorylation, however, required additional co-factors to induce gene activation. The distinct signaling pathways involved in CREB activation in the neonatal heart at 1 and 7 days of age may therefore lead to an age-specific induction of different genes.

\section{REFERENCES}

1. Sun LS, Quamina A 2003 Perinatal cocaine exposure stimulates the expression and activation of CREB in the neonatal rat heart. Pediatr Res 53:500-506

2. Finkbeiner S, Tavazoie SF, Maloratsky A, Jacobs KM, Harris KM, Greenberg ME 1997 CREB: a major mediator of neuronal neurotrophin responses. Neuron 19:10311047

3. Ginty DD, Bonni A, Greenberg ME 1994 Nerve growth factor activates a Rasdependent protein kinase that stimulates c-fos transcription via phosphorylation of CREB. Cell 77:713-725

4. Impey S, Obrietan K, Storm DR 1999 Making new connections: role of ERK/MAP kinase signaling in neuronal plasticity. Neuron 23:11-14

5. Perkinton MS, Sihra TS, Williams RJ $1999 \mathrm{Ca}(2+)$-permeable AMPA receptor induce phosphorylation of cAMP response element-binding protein through a phosphatidylinositol 3-kinase-dependent stimulation of the mitogen-activated protein kinase signaling cascade in neurons. J Neurosci 19:5861-5874

6. Vanhoutte P, Barnier JV, Guibert B, Pages C, Besson MJ, Hipskind RA, Caboche J 1999 Glutamate induces phosphorylation of Elk-1 and CREB, along with c-fos activation, via an extracellular signal-regulated kinase-dependent pathway in brain slices. Mol Cell Biol 19:136-146

7. Frodin M, Gammeltoft S 1999 Role and regulation of $90 \mathrm{kDa}$ ribosomal S6 kinase (RSK) in signal transduction. Mol Cell Endocrinol 151:65-77

8. Zhao Y, Bjorbaek C, Moller DE 1996 Regulation and interaction of pp90(rsk) isoforms with mitogen-activated protein kinases. J Biol Chem 271:29773-29779
9. Takahashi E, Abe J, Berk BC 1997 Angiotensin II stimulates p90rsk in vascular smooth muscle cells. A potential $\mathrm{Na}(+)-\mathrm{H}+$ exchanger kinase. Circ Res 81:268-273

10. Xing J, Ginty DD, Greenberg ME 1996 Coupling of the RAS-MAPK pathway to gene activation by RSK2, a growth factor-regulated CREB kinase. Science 273:959-963

11. Sadoshima J, Qiu Z, Morgan JP, Izumo S 1995 Angiotensin II and other hypertrophic stimuli mediated by $\mathrm{G}$ protein-coupled receptors activate tyrosine kinase, mitogenactivated protein kinase, and 90-kD S6 kinase in cardiac myocytes. The critical role of $\mathrm{Ca}(2+)$-dependent signaling. Circ Res 76:1-15

12. Seko Y, Takahashi N, Tobe K, Ueki K, Kadowaki T, Yazaki Y 1998 Vascular endothelial growth factor (VEGF) activates Raf-1, mitogen-activated protein (MAP) kinases, and S6 kinase (p90rsk) in cultured rat cardiac myocytes. J Cell Physiol 175:239-246

13. Xu YJ, Ouk Kim S, Liao DF, Katz S, Pelech SL 2000 Stimulation of 90- and 70-kDa ribosomal protein $\mathrm{S} 6$ kinases by arginine vasopressin and lysophosphatidic acid in rat cardiomyocytes. Biochem Pharmacol 59:1163-1171

14. Mayr B, Montminy M 2001 Transcriptional regulation by the phosphorylationdependent factor CREB. Nat Rev Mol Cell Biol 2:599-609

15. Shaywitz AJ, Greenberg ME 1999 CREB: a stimulus-induced transcription factor activated by a diverse array of extracellular signals. Annu Rev Biochem 68:821-861

16. Lin RZ, Chen J, Hu ZW, Hoffman BB 1998 Phosphorylation of the cAMP response element-binding protein and activation of transcription by alpha1 adrenergic receptors. J Biol Chem 273:30033-30038

17. Soderling TR 1999 The Ca-calmodulin-dependent protein kinase cascade. Trends Biochem Sci 24:232-236

18. Sheng M, Thompson MA, Greenberg ME 1991 CREB: a Ca(2+)-regulated transcription factor phosphorylated by calmodulin-dependent kinases. Science 252:1427-1430

19. Cammarota M, Bevilaqua LR, Dunkley PR, Rostas JA 2001 Angiotensin II promotes the phosphorylation of cyclic AMP-responsive element binding protein (CREB) at Ser133 through an ERK1/2-dependent mechanism. J Neurochem 79:1122-1128

20. Deak M, Clifton AD, Lucocq LM, Alessi DR 1998 Mitogen- and stress-activated protein kinase-1 (MSK1) is directly activated by MAPK and SAPK2/p38, and may mediate activation of CREB. EMBO J 17:4426-4441

21. Iordanov M, Bender K, Ade T, Schmid W, Sachsenmaier C, Engel K, Gaestel M, Rahmsdorf HJ, Herrlich P 1997 CREB is activated by UVC through a p38/HOG-1dependent protein kinase. EMBO J 16:1009-1022

22. Hansen TV, Rehfeld JF, Nielsen FC 1999 Mitogen-activated protein kinase and protein kinase A signaling pathways stimulate cholecystokinin transcription via activation of cyclic adenosine 3',5'-monophosphate response element-binding protein. Mol Endocrinol 13:466-475

23. Xing J, Kornhauser JM, Xia Z, Thiele EA, Greenberg ME 1998 Nerve growth factor activates extracellular signal-regulated kinase and p38 mitogen-activated protein kinase pathways to stimulate CREB serine 133 phosphorylation. Mol Cell Biol 18:1946-1955

24. Du K, Montminy M 1998 CREB is a regulatory target for the protein kinase Akt/PKB. J Biol Chem 273:32377-32379

25. Sassone-Corsi P 1995 Transcription factors responsive to cAMP. Annu Rev Cell Dev Biol 11:355-377

26. Davis S, Vanhoutte P, Pages C, Caboche J, Laroche S 2000 The MAPK/ERK cascade targets both Elk-1 and cAMP response element-binding protein to control long-term potentiation-dependent gene expression in the dentate gyrus in vivo. J Neurosci 20:4563-4572

27. Sato-Bigbee C, Pal S, Chu AK 1999 Different neuroligands and signal transduction pathways stimulate CREB phosphorylation at specific developmental stages along oligodendrocyte differentiation. J Neurochem 72:139-147

28. Funakoshi Y, Ichiki T, Takeda K, Tokuno T, Iino N, Takeshita A 2002 Critical role of cAMP-response element-binding protein for angiotensin II-induced hypertrophy of vascular smooth muscle cells. J Biol Chem 277:18710-18717

29. Bohm M, Moellmann G, Cheng E, Alvarez-Franco M, Wagner S, Sassone-Corsi P, Halaban R 1995 Identification of p90RSK as the probable CREB-Ser133 kinase in human melanocytes. Cell Growth Differ 6:291-302

30. Takeishi Y, Huang Q, Abe J, Che W, Lee JD, Kawakatsu H, Hoit BD, Berk BC, Walsh RA 2002 Activation of mitogen-activated protein kinases and p90 ribosomal S6 kinase in failing human hearts with dilated cardiomyopathy. Cardiovasc Res 53:131-137

31. Kim SO, Irwin P, Katz S, Pelech SL 1998 Expression of mitogen-activated protein kinase pathways during postnatal development of rat heart. J Cell Biochem 71:286301

32. Nakajima T, Fukamizu A, Takahashi J, Gage FH, Fisher T, Blenis J, Montminy MR 1996 The signal-dependent coactivator CBP is a nuclear target for pp90RSK. Cell $86: 465-474$

33. Wang Z, Zhang B, Wang M, Carr BI 2003 Persistent ERK phosphorylation negatively regulates cAMP response element-binding protein (CREB) activity via recruitment of CREB-binding protein to pp90RSK. J Biol Chem 278:11138-11144

34. Mayr BM, Canettieri G, Montminy MR 2001 Distinct effects of cAMP and mitogenic signals on CREB-binding protein recruitment impart specificity to target gene activation via CREB. Proc Natl Acad Sci U S A 98:10936-10941 